\title{
Diagnóstico político de la evaluación en Argentina
}

\author{
Carlos Pérez Rasetti \\ Universidad Nacional de La Patagonia Austral
}

\section{Resumen}

Desde la implantación de la evaluación y acreditación en Argentina los objetivos de las políticas iniciales se fueron diluyendo, diversificando, transformando por acción de las propias políticas públicas y las impulsadas por los colectivos de actores implicados. Hoy, cada una de las cuatro funciones sustantivas de la Coneau levaluación de proyectos de nuevas instituciones, evaluación externa de instituciones en funcionamiento, acreditación de carreras de grado -pregrado para otros países- y acreditación de carreras de posgradol presenta una significación política dispar que este artículo trata de caracterizar y explicar.

Palabras clave: Evaluación. Acreditación. Instituciones universitarias. 


\section{Political diagnosis of evaluation in Argentina}

Since the implementation of evaluation and accreditation processes in Argentina, the aims of the initial policies began being diluted, diversified and transformed by public policies themselves, and some due to pressures from groups of actors involved. Today, each one of CONEAU's four substantive functions levaluation of projects from new institutions, external evaluation of institutions in operation, (foreign) undergraduate and graduate programme accreditation) has a different political purport, which this article aims to characterize and explain.

Keywords: Evaluation. Accreditation. Universities.

\section{Diagnóstico político da avaliação na Argentina}

Desde a implementação da avaliação e do credenciamento na Argentina, os objetivos das políticas iniciais foram se diluindo, diversificando-se, transformando-se pela ação das próprias políticas públicas e as impulsionadas pelos coletivos dos atores implicados. Hoje, cada uma das quatro funções básicas da CONEAU lavaliação de projetos de novas instituições, a avaliação externa das instituições em funcionamento, o credenciamento de cursos de graduação e pós-graduaçãol possuem significados políticos díspares que este artigo tenta caracterizar e explicar.

Palavras-chave: Avaliação. Credenciamento. Instituições de Educação Superior. 


\section{Introducción}

La evaluación de instituciones y programas se inició en Argentina en los años noventa por iniciativa estatal y se ha mantenido hasta el presente en el marco de un escenario político que presenta grandes contrastes. La década de los noventa se caracterizó por la implementación en Argentina de políticas de orientación neoliberal o neoconservadoras, inspiradas en el llamado Consenso de Washington. A fines de 1989 asumió la presidencia Carlos Menem, en el marco de una fuerte crisis económica con hiperinflación. La etapa se caracterizó por el recorte de los gastos estatales, la reducción del sector público, la privatización de las empresas estatales y de los servicios públicos, consumándose una decidida retirada del Estado en todos los niveles. En el caso de la Educación Superior, que en Argentina tenía una arraigada tradición de gratuidad la presión del gobierno estuvo orientada, principalmente, a introducir una dinámica de mercado. Se habilitó la creación de nuevas universidades privadas, que estaba suspendida desde 1973, se autorizó a las universidades estatales a percibir aranceles por los estudios y por otros servicios y se redujeron los presupuestos universitarios. La implementación de la evaluación estaba pensada como una maniobra de pinzas, un modo de exponer las diferencias de calidad entre las universidades y entre sus carreras, incentivando la competencia y ejerciendo presión para que tuvieran que buscar recursos para mejorar en el mercado.

Cuando Menem dejó el gobierno, la economía argentina ya llevaba tres años de recesión. Lo sucedió, en diciembre de 1999. Fernando de la Rua que, más allá de sus discrepancias discursivas con el modelo anterior, no atinó a cambiar las políticas dominantes y fue arrastrado por la situación económica y social en decidido deterioro. Todo esto culminó en diciembre de 2001 con el sistema bancario colapsado, el Estado en cesación de pagos y la desocupación en niveles superiores al 30\%. Un estallido social, con manifestaciones y protestas en todo el país, represión y más de 20 muertos determinó la renuncia del presidente.

Luego de una serie de presidentes provisionales, en 2003 asumió Carlos Kirchner e inició una política de signo inverso a la que caracterizó a los años noventa; esta política continúa con los gobiernos de Cristina Fernández de Kirchner hasta la actualidad. El protagonismo del Estado fue la marca de esta etapa, con políticas sociales activas, una economía heterodoxa con fuerte promoción de las actividades económicas de tipo productivo y una progresiva recuperación del empleo. En la política universitaria también los cambios fueron de 180 grados. Lo primero que se hizo fue devolver a las universidades estatales los montos presupuestarios no devengados en los años anteriores, luego se inició una recuperación de los salarios y de la inversión tanto en infraestructura como en equipamiento y recursos humanos. En cuanto a la evaluación los cambios fueron de contexto, porque cambiaron las políticas en las que la evaluación y, especialmente la acreditación de carreras de grado, están insertas. Ya, en ningún caso, la evaluación tiene como 
finalidad la exposición de diferencias ni la de generar una presión a favor de una dinámica de mercado. Las particularidades se analizarán en cada caso.

Con todo, a pesar de los cambios en la legislación educativa, la Ley de Educación Superior de 1995 sigue vigente. Este es el marco en el que realizamos este diagnóstico político de la evaluación en Argentina; el paso de estos instrumentos de un escenario político a otro y los cambios de sentido que ha implicado esta deriva.

\section{La implementación de la evaluación: la Coneau}

La Secretaría de Políticas Universitarias, dependencia creada en 1993 para jerarquizar el área responsable de la educación superior en el Ministerio de Educación e impulsar reformas en el marco de las políticas económicas neoliberales, lanzó dos estrategias paralelas con el objetivo de implementar e institucionalizar una serie de prácticas evaluativas. Por un lado retomó el debate sobre la introducción en el sistema universitario de la evaluación institucional que el gobierno había iniciado en 1991. En ese año llegó a un acuerdo con las universidades nacionales agrupadas en el Consejo Interuniversitario Nacional (CIN) el desarrollo de una metodología de evaluación institucional en el marco del llamado "Proyecto 06: Fortalecimiento de la gestión y Coordinación Universitaria" que financió el Banco Mundial (Paviglianitti et alia: 1996, p. 39). Por otro lado, creó por decreto la Comisión de Acreditación de Posgrados (CAP) en 1994, para evaluar las carreras del cuarto nivel. La CAP funcionaba como opción de acreditación voluntaria y estaba dirigida exclusivamente a los doctorados y las maestrías académicas, dejando de lado las "profesionales". A partir de la aprobación de la Ley de Educación Superior N²4.521, a mediados de 1995, la evaluación de posgrados ingresa definitivamente en la normativa de nuestro país, con carácter obligatorio y extendida a todas las carreras de posgrado, sean estas de orientación académica o profesional, y en sus tres titulaciones: especializaciones, maestrías y doctorados. Pero esta operación de fortalecimiento institucional y ampliación de funciones de una Agencia estatal de evaluación, se da en un contexto más amplio de incorporación de nuevas direcciones de políticas de evaluación. La Ley aprobada incorpora un nuevo organismo, la Comisión Nacional de Evaluación y Acreditación Universitaria (Coneau), para encargarle la evaluación de los proyectos de nuevas universidades, de carreras de grado (pregrado, en otros países) declaradas previamente de interés público, la evaluación de instituciones y, como ya dijimos, de carreras de posgrado.

De esas cuatro grandes funciones no solo la evaluación de carreras de posgrado y la evaluación institucional tenían algún tipo de antecedente en las políticas del gobierno. Ya en 1993, a través del Decreto 2.330 de ese año, se estableció un 
mecanismo para el análisis técnico de los proyectos de nuevas universidades, en una operación de translación de la decisión política a instancias técnico/ evaluativas. Esa operación se perfeccionó mediante las disposiciones de la Ley que remite ese rol, que cumplían los funcionarios del Ministerio, a la Coneau. La ley profundiza radicalmente el papel de la instancia técnico/evaluativa por sobre la decisión política ya que la tornó vinculante para el Poder Ejecutivo, en los casos en que la Coneau emite dictamen negativo.

En cuanto a la Evaluación Institucional, el debate fue intenso en foros académicos y en los organismos que nuclean a las universidades públicas (Consejo Interuniversitario Nacional)) y privadas (Consejo de Rectores Universidades Privadas); pero el CIN se expresó en contra de la conveniencia de aplicar la metodología resultante del proyecto 06 (Paviglianitti et alia, 1996, p.39) y en el debate por la Ley de Educación Superior la mayoría de las universidades nacionales se expresaron oficialmente en contra de la evaluación institucional externa que se estaba proponiendo (Castro, 2005) y que, finalmente, se aprobó.

Este trabajo tratará de repasar la deriva de estas políticas de evaluación implementadas a partir de la Ley de Educación Superior (24.521) de 1995, que crea la Coneau y la establece como organismo responsable principal de la implementación de las políticas de evaluación. Con todo, debe notarse que existen también otras evaluaciones, como las de programas de educación a distancia y de sedes universitarias a radicarse fuera de la propia jurisdicción geográfica, que están encargadas al Ministerio de Educación y a los Consejos Regionales de Planificación de la Educación Superior, respectivamente. Centraremos nuestra atención aquí, en las evaluaciones que son funciones de la Coneau.

\section{El sentido de la evaluación en la Ley de Educación Superior}

La Ley de Educación Superior (LES) se propuso incidir en el cuasi mercado de la Educación Superior Argentina incrementando los niveles de autonomía presupuestaria de las universidades estatales e implementando la evaluación externa para todas las instituciones (también para las privadas) y la publicidad de los resultados de las evaluaciones. De esta manera, las universidades debían ingresar en las reglas de un mercado nuevo para nuestra sociedad, un mercado inducido artificialmente por una política pública que entendía que era la competencia la que asignaba, más eficientemente, pertinencia a las acciones de las universidades y la que se convertiría en impulsora de la calidad y la excelencia académicas.

La evaluación y la acreditación universitarias que se han hecho práctica corriente en todo el mundo, no tienen siempre la misma significación, ni persiguen los mismos objetivos políticos. En los años 90' tomó fuerza en algunos países la instrumentación de estas prácticas como modo de forzar a las universidades a 
entrar en el mercado. La evaluación y/o la acreditación funcionaban como un modo de exponer la situación de las instituciones y carreras frente a la sociedad, de calificarlas para orientar el mercado tanto de estudiantes, como de las empresas que buscan profesionales. En esta concepción se instauró el Provâo brasilero durante el gobierno de Fernando Henrique Cardoso ${ }^{1}$ y la Ley de Educación Superior (LES) de 1995 en la Argentina que gobernaba Carlos Menem². En la LES se establece la obligatoriedad de la Evaluación Institucional y de la Acreditación de las carreras de grado de interés público. Al mismo tiempo se habilita a las instituciones a generar recursos propios arancelando sus servicios incluyendo los estudios de grado y postgrado. Complementariamente se establece la prohibición para el Estado de reducir los aportes económicos en función de los ingresos generados. Esta cláusula es clave porque se constituye en un estímulo para la generación de ingresos adicionales. En la negociación que tuvo lugar durante el tratamiento de la ley en la Cámara de Diputados, se prohibió que los fondos recaudados por aranceles impuestos a los estudios de grado fueran utilizados para gastos corrientes y se señaló taxativamente como destino, el financiamiento de acciones de apoyo a los estudiantes (Nosiglia; Marquina, 1996, p.101). Este aspecto, levemente incoherente con los fines de la ley, no desvirtúa el conjunto de los instrumentos que apuntan al mercado. De hecho, la concepción que subyace era que en la Argentina los problemas de calidad del sistema universitario se debían a la ausencia de un mercado estricto. Para esa manera de ver las cosas, las universidades nacionales funcionan sólo con aportes estatales que resultaban insuficientes y utilizan de manera ineficaz esos fondos provenientes del Tesoro Nacional. Subsidian a estudiantes que pertenecen a familias con recursos y compiten deslealmente con las universidades privadas que, en ese contexto, no pueden cobrar los aranceles que debieran y, por lo tanto, terminan también desfinanciadas. A esto se agregaba el planteo de que las universidades nacionales generarían actividades mediocremente avaladas por el subsidio estatal, sin planificación y sin necesidad de sujetarse a parámetros de demanda. Todo el sistema administra títulos habilitantes con respaldo del estado y no existen en el mercado indicadores que permitan distinguir distintos niveles de calidad ${ }^{3}$. Es decir, no hay competencia, y por lo tanto, no hay estrictamente hablando, mercado.

La introducción de la Comisión Nacional de Evaluación y Acreditación Universitaria en la LES y de los sistemas de Evaluación y Acreditación que ésta tiene a su cargo, apuntaban a establecer la calidad de las instituciones y de sus ofertas (carreras de grado de interés público y postgrados) y publicitarlas. Este

1. El Examen Nacional de Cursos (Provão) era un sistema de evaluación y de jerarquización de las carreras claramente destinado a calificarlas de cara al mercado.

2. El Examen Nacional de Cursos (Provão) era un sistema de evaluación y de jerarquización de las carreras claramente destinado a calificarlas de cara al mercado.

3. La fundación F.I.E.L. expuso estas opiniones que luego expresó brevemente en el Ministerio de Economía Ricardo López Murphi. (2001) 
factor es fundamental para generar la competencia en un régimen de títulos en el que la habilitación profesional está ligada al diploma académico e, incluso, lo desvirtúa proyectando la imagen habilitante en carreras que forman en disciplinas no relacionadas con profesiones reguladas por el estado. La LES prevé un mecanismo adicional para corregir la falta de dirección del aporte estatal y ponerlo en línea con esta concepción inductora de reglas de mercado. El artículo 58 de la LES establece que en la distribución del aporte financiero para el sostenimiento de las instituciones universitarias nacionales "se tendrán especialmente en cuenta indicadores de eficiencia y equidad".

Esto es, las universidades y sus ofertas están obligadas a la competencia para obtener sus recursos, provengan estos del Tesoro Nacional o del Mercado. Expuestas por los resultados de las evaluaciones tendrán que generar recursos propios buscando en el mercado aportes adicionales para mejorar su calidad, porque lo que el Estado no puede negarles en razón de esos ingresos, si puede restarlo o no incrementarlo a causa de los malos resultados.

\section{Desplazamientos de sentido en la implementación de la evaluación}

En cuanto a la implementación del sistema de evaluación y acreditación, la propia Coneau, encargada de llevar a cabo las evaluaciones previstas en la legislación, empezara a cambiar los objetivos en su instrumentación. Por un lado le quitó de hecho obligatoriedad a la Evaluación Institucional, permitiendo que las universidades eligieran el momento en que estaban decididas a encararla, introduciendo incluso una modalidad de acuerdos voluntarios para algo que en la ley es un deber periódico. El Ministerio nunca ejerció algún poder de policía sobre las instituciones reticentes a cumplir el mandato legal, que durante los primeros años fueron mayoría. La Coneau tampoco diseñó criterios rígidos y estableció que toda evaluación institucional se hiciera teniendo como referencia el modelo que la universidad se había propuesto para sí misma. En cuanto a la acreditación de carreras de grado, habilitó una instancia intermedia, la “acreditación por tres años con compromisos de mejoramiento" que trasladó el resultado del proceso de la calificación (acredita/no acredita) al mejoramiento, ya que el $80 \%$ de las carreras que pasaron por el proceso están en esa nueva categoría que apuesta a la mejora. (Krostch, 2002)

La LES, por la resistencia y la acción de la comunidad universitaria, incluso expresándose a través de los organismos de coordinación y evaluación que la ley en cuestión instituyó, no logró privatizar o meter "en mercado" a la universidad pública y, al mismo tiempo, convirtió pretendidos instrumentos de política neoliberal en un excelente dispositivo para elaborar diagnósticos y propuestas de mejoramiento de las carreras. 
Este proceso pone en evidencia que los sistemas de evaluación no son instrumentos de un solo sentido. Más bien adquieren su sentido según la política pública en la que están insertos. El proceso de implementación en nuestro país inició un corrimiento de las políticas referidas a la evaluación, que primero fue el resultado de una práctica del sistema universitario, de las instituciones y de los actores y tuvo en los gobiernos una respuesta prudente que se expresó en una disminución de la presión por el cumplimiento de la ley y finalmente la renuncia, a partir de 2003 a esas políticas de presión pro mercado. Téngase en cuenta que nunca le fue quitado el reconocimiento nacional a una carrera de grado no acreditada, ni se tradujo en algún castigo concreto la falta de adhesión a los procesos de evaluación institucional para las universidades nacionales o privadas con reconocimiento definitivo. El sentido de la evaluación y de la acreditación cambió para convertirse, aun con sus defectos, en un sistema de diagnóstico y diseño de estrategias de mejoramiento.

Pero a partir del año 2002 el Poder Ejecutivo empieza a dar señales de lo que será un cambio profundo de política y la adhesión a otra concepción de la relación de la universidad con el Estado. En ese año se asignaron algo menos de $\$ 900.000$ (apenas unos 200 mil dólares, en ese momentol para el financiamiento de los Planes de Mejoras de las carreras de Medicina que habían pasado por el proceso de acreditación (Pugliese, 2003; Broto, 2003). Esto sucedía en el marco de la crisis cuyo punto abismal no había cumplido aún un año y mientras se procuraba reparar las deudas generadas por las sub ejecuciones presupuestarias de los años anteriores. Era una señal, una operación simbólica que pretendía anunciar una política para la eventual recuperación.

A fines del año 2003 la Secretaría de Políticas Universitarias presentó en las Jornadas de Reflexión sobre la Educación Superior organizadas por el CIN en Horco-Molle, en la sede de la Universidad Nacional de Tucumán, un documento de políticas que fue discutido por los rectores quienes a su vez generaron su propio texto como respuesta (CIN 2003). Así se inició un diálogo que enmarcaría el desarrollo posterior de este cambio de política. Se pactaba una transformación en las relaciones entre las universidades nacionales y el Estado que suponía una práctica de corresponsabilidad y consenso. El Estado se hacía cargo de la función social de la educación universitaria, abandonaba el rol de evaluador descomprometido, iniciaba un diálogo con las universidades en el que la discusión de las políticas asumía a las universidades como sujeto y objeto de políticas públicas. Las universidades ponían en la mesa sus propias prioridades y acordaban discutir prioridades nacionales y regionales. Se comenzaba a desandar la desconfianza que signó las relaciones durante el período anterior, en el que la resistencia se había hecho cultura institucional. 


\section{El giro de sentido se completa en la acreditación de carreras de grado}

Enlaacreditación decarreras degradotuvounalto gradodeadhesión. Participaron casi todas las carreras o programas convocados y todas las universidades, pero es cierto que, durante los primeros años la Universidad de Buenos Aires mantuvo al margen su carreras de Medicina, primero, y de Ingeniería después, siempre al amparo de un fallo judicial firme que la excluyó de los alcances de la LES en los artículos relacionados con la Coneau y los procesos de evaluación y acreditación. La primera carrera de grado perteneciente a la Universidad de Buenos Aires que ingresó a los procesos de acreditación fue la de Ingeniería Agronómica pero lo hizo mediante un procedimiento lateral, presentándose al Mecanismo Experimental de Acreditación del Mercosur (MEXA) que, aunque lo ejecutaba la Coneau, no se realizaba, al menos estrictamente, en cumplimiento del artículo 43 de la LES sino en el marco de acuerdos regionales. Posteriormente, la Coneau proveyó, de oficio, las acciones de evaluación complementarias que convalidaron la evaluación MEXA de Agronomía de la UBA en términos de acreditación nacional. Para esa época también se verificó la rebeldía de alguna que otra unidad académica (facultad) de universidades que habían ya participado con carreras de otras áreas. El caso más notorio fue el de la Universidad Nacional del Comahue ya que la cuestión terminó en un conflicto más amplio que paralizó el funcionamiento institucional por un largo período.

Con mejor situación de la economía y de las finanzas del Estado y establecida por el gobierno condición prioritaria para el desarrollo de la educación y de la educación superior en particular, el aporte simbólico realizado a las primeras 9 carreras de medicina que pasaron por los procesos de acreditación se convirtió en un Programa de Calidad que comenzó con las carreras de ingeniería. El desarrollo de la primera versión Proyecto de Mejoramiento de la Enseñanza de la Ingeniería (PROMEI I) durante los años 2004 y 2005, que ya contaba con un financiamiento sustantivo (más de 68 millones de pesos) para apoyar los planes de mejoramiento de las carreras de ingeniería que habían pasado por el proceso de acreditación, marcó el momento de consolidación de esta política y cambió radicalmente el sentido de la acreditación. El Proyecto tenía características que definen la nueva concepción, apartada ya definitivamente de la que impulsaba la LES. Los fondos no eran competitivos, no hubo premios ni castigos, se asignaron a todas las carreras cualquiera sea el resultado obtenido en la acreditación. Para las carreras que alcanzaron el mejor nivel de acreditación el financiamiento apuntalaba la búsqueda de la excelencia, para las que se comprometieron a implementar estrategias de mejoramiento está destinados a sostener esas iniciativas y en el caso de las que no acreditaron, el proyecto se apoya en las fortalezas que se hubieren identificado y desde ahí se apoya la construcción de condiciones que permitan el desarrollo de la calidad posible. 
El primer Proyecto trabajó sobre prioridades establecidas por la Secretaría de Políticas Universitarias (SPU) a partir de los estudios realizados sobre los resultados de la evaluación de las carreras de ingeniería, en función de objetivos que estaban delineados en el modelo que los estándares de acreditación proponían y particularizado en un proyecto que cada facultad debía presentar basado en sus compromisos de mejora establecidos en la resolución de acreditación (dictamen de Coneaul o en propuestas de excelencia. Los ejes estaban puestos en el fortalecimiento de la formación básica, en la atención especializada a los alumnos ingresantes (atacar la importante deserción), el fortalecimiento de la investigación, de las dedicaciones exclusivas, pero orientadas a profesores posgraduados o con experiencia en investigación, y la formación de posgrado (también muy escasa en las facultades de ingeniería de Argentinal. Uno de los aspectos más novedosos de este programa de financiamiento de mejoras fue la incorporación de ítems con financiamiento recurrente, destinado, especialmente, a sostener presupuestariamente las incorporaciones de recursos humanos académicos.

De ahí en más se institucionalizaron los proyectos de mejoramiento en un Programa de Calidad que ligó la acreditación de carreras de grado de interés público con el financiamiento de las medidas de mejoramiento surgidas de esos diagnósticos. De ahí en más "Proyecto de Mejoramiento" para cada etapa de la acreditación de carreras de ingeniería y luego para las carreras de agronomía, las de farmacia y bioquímica, agronomía, veterinaria, arquitectura, ingeniería en recursos naturales y forestal, odontología, medicina, y así siguiendo.

De esta manera queda completado el giro de sentido que la política le dio a la acreditación. La descripción de este desplazamiento me parece más fácil hacerla utilizando una clasificación de modelos de relación entre políticas públicas y sistemas de evaluación que desarrollé en un trabajo anterior (Pérez Rasetti, 2010). Dos de los modelos propuestos corresponderían, en mi opinión, a la acreditación de grado en el marco de lo propuesto por la ley (modelo de 'calificación' para los años novental y a la situación construida a partir de 2003 ('planificación'). En el primer caso se caracteriza una relación en la que la política utiliza a la evaluación como calificadora de productos para el mercado; se limita a aportar descripción de las carreras y por lo tanto, a jerarquizarlas públicamente produciendo una dinamización del mercado. En el caso de Argentina, donde no había un mercado de educación superior pero se lo quería impulsar, era necesario que la acreditación fuera obligatoria para que ninguna carrera pudiera evitar su exposición pública en una sociedad acostumbrada al respaldo estatal igualador para las carreras universitarias que administran titulaciones de carácter público y que son habilitantes para el ejercicio profesional, cuando es el caso. 


\section{Proyectos de nuevas instituciones: la política de evaluación reemplaza a la política}

Tanto para la creación de universidades nacionales como para la autorización de las privadas, el Poder Ejecutivo se retira de la política en el gesto de confiar la política a la ley y el objetivo de esa política, la calidad, a la técnica y a la competencia y al mercado, el juicio de pertinencia. En la LES se definen instrumentos que transfieren parcialmente la decisión de la órbita estrictamente política a la esfera técnica. La intención parece ser, en ambos casos, restrictiva; subyace un juicio crítico sobre el modo que adquirió la expansión del sistema mediante el procedimiento de creación de nuevas instituciones y se trata de encausar y, probablemente también de contener, la tendencia expansiva en esta línea.

En el caso de las instituciones privadas es evidente la intención de sujetar la autorización sólo al juicio de calidad, desistiendo de cualquier consideración de pertinencia; para las instituciones públicas se propicia el juicio de calidad pero al mismo tiempo se propone la intervención, aunque no vinculante (no podía ser de otro modol del sistema, histórica y naturalmente reticente a la expansión y a la competencia, especialmente en épocas de restricciones presupuestarias como las que se vivían en la década de los noventa.

Haciendo algo parecido a un balance, para el caso de las instituciones privadas podría afirmarse que la política restrictiva por vía de la técnica evaluativa alcanzó cierto éxito. Solo comparando el número de instituciones autorizadas antes y después de la sanción de la LES se nota su efectividad. Bajo el régimen previo en cualquiera de sus dos etapas: desde 1990 hasta diciembre de 1993 en que se dictó el decreto 2330/93, se autorizaron catorce (14) universidades y un (1) instituto universitario; a partir del dictado de ese decreto y hasta la vigencia de la LES en agosto de 1995, se autorizaron otras siete (7) universidades. En los quince años que han pasado desde que se puso en funcionamiento la Comisión Nacional de Evaluación y Acreditación Universitaria (Coneau), se han autorizado apenas catorce (14) institutos universitarios y cuatro (4) universidades, con lo que se pasó de un ritmo de algo más de cuatro autorizaciones por año a poco más de una. Esta reducción a un cuarto de la cantidad de autorizaciones anuales no se debe a la ausencia de proyectos; en sus años de existencia la Coneau lleva considerados unos cien Proyectos Institucionales de nuevas instituciones privadas.

En el caso del subsector universitario nacional la evaluación de los Proyectos Institucionales por parte de la Coneau no tiene implicancias en el proceso de creación (que se hace por ley e incumbe, por lo tanto, al Congreso y al Poder Ejecutivol sino en el proceso de implantación de las nuevas instituciones, en el que le compete la aprobación del Proyecto Institucional propuesto por la autoridad a cargo de la implantación de la nueva universidad. 


\section{Problemas políticos en la acreditación de carreras de posgrado}

Lo primero que se puede señalar desde la perspectiva política es que, desde la creación de la CAP (Comisión de Acreditación de Posgrados) 1994, la única política de posgrado evidente, consistente y continuada, se ha resuelto a través de la evaluación. Ahora bien, ¿cuál es la política detrás del instrumento de la evaluación? La primera etapa, instrumentada a través de la CAP, nos muestra de manera bastante transparente las claves del sentido de esta política ya que en ese comienzo están los elementos cuya institucionalización se fortalece después con la Ley de Educación Superior de 1995 y la implementación de la Coneau.

La creación de la CAP se da en el marco de un fuerte crecimiento de la oferta de carreras de posgrado (especialmente en la modalidad de Maestría) aranceladas y ofrecidas tanto por universidades públicas como privadas. Es evidente que se comienza a conformar un mercado alrededor de la formación de posgrado y que las instituciones universitarias actúan de manera activa y dinámica en su conformación, mostrando un accionar totalmente diverso del que manifestaban en el campo de las carreras de grado (pregrado). Ese mercado tiene su centro en la oferta de maestrías ya sea porque son la novedad, las que más crecen en demanda (por efecto del credencialismo en aumento y las facilidades que prometen por su modalidad escolarizadal y, por lo tanto, en cantidad, como porque su precio comienza a segmentarse de manera notoria de acuerdo a los sectores de demanda. Los doctorados ocupan, en cambio, un espacio marginal en el mercado de carreras de posgrado. (Barsky, 1996; García de Fanelli, 1996, 2000b; Guerrini et alia, 2004)

La evaluación de la CAP viene entonces a intervenir en la dinámica de este proceso de expansión con las siguientes características: se trata de procesos de acreditación voluntaria, la evaluación incluye la jerarquización de las carreras en tres categorías, se vincula la evaluación con líneas de financiamiento y el proceso de acreditación sólo comprende a los doctorados y maestrías académicas. La primera observación que surge del análisis es que la política impulsada desde la SPU segmenta el mercado de posgrado en dos grupos: las carreras propiamente académicas y las profesionales y que no incluye a este segundo segmento en la evaluación de la CAP. Es decir, la política considera que no es necesario intervenir en el mercado de la oferta de carreras de posgrado profesionales justamente porque, al estar directamente orientada a un mercado competitivo, se trabajó con la hipótesis de que el propio mercado se encargaría de discriminar la oferta de acuerdo a los niveles de 'calidad', impulsado por la competencia; y que también regularía la proliferación de carreras, descartando a las ofertas de menor interés o calidad; es decir, el mercado también se encargaría de la 'pertinencia'.

En el caso de las carreras de orientación académica, dirigidas por lo tanto a los profesores universitarios y a los investigadores, campo en el cual el mayor empleador es el Estado y, por lo tanto, no reúne realmente las condiciones de un 
mercado estrictamente hablando, la CAP debía discriminar por calidad y generar la dinámica de competencia. La intervención del Estado se orienta, entonces, a promover condiciones de mercado en el sector, alentando la competencia mediante la discriminación de niveles de calidad; esto es, carreras acreditadas, no acreditadas y no presentadas a acreditación; carreras acreditadas con diversas categorías. El carácter voluntario del procedimiento de acreditación es típico de este modelo ya que, mientras previene críticas desde la perspectiva autonómica, logra generar presión sobre las carreras no acreditadas en la medida en que aquellos que se saben mejores, recurrirán a esta modalidad para legitimar su calidad y despegarse del resto. La esperable participación de los mejores produce también un efecto sobre la propia acreditación, trasladando el prestigio previo de las carreras al procedimiento, legitimándolo de esa manera.

La aprobación de la Ley 24.521 de Educación Superior en 1995, a un año de iniciada la experiencia de la CAP, introduce dos modificaciones importantes en la evaluación de carreras de posgrado (art. 46). Por un lado, extiende el procedimiento a todas las carreras de posgrado, eliminando la segmentación que hacía la CAP, y se establece su obligatoriedad, frente al carácter voluntario que caracterizó la etapa de aquella primera agencia.

Este ajuste de la política de evaluación de posgrado, que es en realidad una política para dinamizar un mercado de posgrado en la Argentina, resulta de la necesidad de acelerar el proceso. Por un lado el mercado (la demanda) es tan grande que la oferta no llega a una efectiva competencia más que en algunos niveles y en algunos campos muy específicos, pero dejando grandes segmentos disponibles para ofertas sin calificación. En este fenómeno influye fuertemente la tradición universitaria argentina de títulos oficiales, es decir, respaldados por el Estado y habilitantes para el ejercicio profesional, en el caso del grado. Más allá de que las carreras de posgrado no "habilitan" para ningún ejercicio profesional, esta arraigada cultura que legitima un diploma que cuenta con el respaldo explícito del Estado, contribuye a que todo título de posgrado tenga su valor en el mercado (sea apreciado) independientemente de la intervención de mecanismos nuevos de legitimación, no instalados aún del todo en el imaginario social, como la acreditación.

La obligatoriedad y la extensión del procedimiento a todas las carreras vienen de esta manera a constituirse en factores de mayor presión, pero dentro de la misma política de contribuir a la conformación de un mercado de carreras de posgrado. Se mantiene, sin embargo, la voluntariedad para la categorización de las carreras, porque obligadas todas a pasar por el proceso de acreditación, es decir, instalada la primera calificación (acredita/no acredital en el sistema de posgrado, lo demás vendría, ahora sí, de la dinámica competitiva. En 1997 se profundiza esta política mediante el dictado de la Resolución Ministerial 1168, que interpreta la obligatoriedad establecida en la ley como aplicable también a los proyectos de carrera, instalando un procedimiento de acreditación previo al reconocimiento 
oficial, es decir, capaz de actuar sobre la cultura de las titulaciones, incluso de incidir sobre los espacios de innovación.

Esta política se ha mantenido sin cambios hasta la actualidad y, en lo sustantivo, ha gozado del consenso pragmático de las instituciones públicas y privadas y de los actores involucrados. Es cierto que los Consejos Superiores de muchas de las universidades nacionales cuestionaron la constitucionalidad de los artículos de la LES referidos a la evaluación y acreditación, incluidos los referidos a las carreras de posgrado. Esto es, aún resistiendo la intervención Estatal para dinamizar e, incluso, para sanear el mercado de posgrado, debe decirse que las universidades lo adoptaron. La explicación es simple y está en la matriz de pagos elaborada por Camou (2006). El posgrado da prestigio académico al que lo ofrece, al que enseña y al que cursa, pero también significa una fuente de ingresos adicionales para muchos profesores y para las instituciones. Y esto funciona así porque se da en una dinámica de mercado, aunque imperfecto, pero que permite, aún a las conducciones universitarias más comprometidas discursivamente con la gratuidad de los estudios de grado, mantener el posgrado arancelado.

Otra respuesta está esbozada en varios trabajos, pero especialmente en el análisis de Pedro Krostch (2002) sobre la modalidad negociada que caracterizó la implantación de los procesos de evaluación y acreditación en la Argentina. Este proceso no fue homogéneo respecto de cada uno de los procesos que la LES encargó a la Coneau. En el caso de la acreditación de posgrado, la política de mercado, como ya dijimos, contó con la aceptación de la mayor parte de la comunidad universitaria, más allá de los gestos ideológicos o declarativos. Con la excusa de que no existían fondos específicos para generar la nueva oferta de posgrados (maestrías y especializaciones), las universidades estatales se sintieron liberadas del compromiso ideológico de la gratuidad y los arancelaron con la razón o excusa de financiarlos.

Finalmente y para resumir, si la única política para el posgrado es la acreditación, la realidad es que la política se ha dejado en manos del mercado y la acción del Estado es apenas subsidiaria. La asignación de pertinencia queda también en manos del mercado, pero de un mercado que ni siquiera resulta estricto por las distorsiones que produce nuestra tradición de títulos con respaldo estatal, reforzada en este caso por la acreditación. De esta manera, el mercado se manifiesta incapaz de regular un sistema de posgrados adecuado para nuestras necesidades. Ni sirvió para regular la expansión excesiva Icon una ritmo tan acelerado que no se compadece con los tiempos de la formación de recursos humanos que ellos mismo requieren) ni para garantizar un acceso igualitario y extenso, a nivel económico o en sentido geográfico.

La política de acreditación deja la asignación de pertinencia en manos del mercado, ya lo dijimos. Esto es, de su dinámica depende la localización de los posgrados, su creación y continuidad, incluso las condiciones en que se replican muchas carreras en otras localizaciones diferentes a aquellas en las que las 
instituciones responsables tienen radicada su capacidad académica. Los problemas que describimos se dan especialmente en el nivel de maestrías, bastante en las especializaciones (con excepción de las médicas), aunque aquí el impacto en la calidad es menor porque no se requiere el insumo más gravoso como es el desarrollo de investigación.

Probablemente se ha esperado de la acreditación de posgrados, que responde al modelo de "calificación" dentro de la clasificación que propusimos oportunamente (Pérez Rasetti 2010), resultados que no está en condiciones de proporcionar si no está inserta dentro del marco de una política más amplia respecto del sistema de posgrado, dotada con instrumentos capaces de corregir las distorsiones que el mercado produce. Pero, a pesar de esto, para la comunidad académica involucrada, la acreditación termina por ser básicamente satisfactoria, ya que proporciona una legitimidad adicional al producto que las carreras ofrecen, que se suma a la que normalmente otorgan el reconocimiento oficial y la validez nacional de los títulos. Así son las cosas, es muy difícil que algo cambie. De hecho, en 2010 se aprobó una nueva resolución de estándares para posgrado (Resolución ME N51/2012) que no cambió la política de acreditación del nivel.

\section{Infalibilidad de la autonomía: sin política en la evaluación institucional}

La Ley de Educación Superior establece que las evaluaciones externas complementan un proceso de autoevaluación llevado a cabo por la propia universidad y que deberá hacerse "en el marco de los objetivos definidos por cada institución". La Comisión Nacional de Evaluación y Acreditación Universitaria, en el momento de iniciar las actividades de evaluación institucional, aprueba un documento denominado: Lineamientos para la Evaluación Institucional (Resolución 094-Coneau-1997) en el que recoge el criterio establecido en la LES para realizar las evaluaciones. En los lineamientos se lee:

Se parte, como antes se dijo, de una premisa básica: el respeto irrestricto a la autonomía universitaria, principio que goza de jerarquía constitucional lart. 75. inc. 19 Constitución Nacional). Este valor subraya el respeto por el proyecto institucional que cada institución determine para sí misma. (Coneau, 1997, p.7)

Cómo puede verse, los Lineamientos traducen "el marco de los objetivos definidos por cada institución" contenido en la Ley, por el concepto de "proyecto institucional". Para la Coneau, en razón de la autonomía, el modelo contra el cual debe realizarse la evaluación institucional de cada universidad es su propio proyecto institucional. Los "Lineamientos" son, obviamente, producto de la época en que fueron redactados. Más que instrumentos metodológicos o direcciones 
para las universidades, constituyen un sistema de garantías que esperaba contribuir -y lo hizo- a superar la polémica recepción que tuvo la Evaluación Institucional, y especialmente la Evaluación Externa, en el sistema universitario argentino, y a habilitar la institucionalización de las evaluaciones Coneau. Más allá de esos objetivos coyunturales, los "Lineamientos" recogen y desarrollan algunos conceptos que están en la Ley de Educación Superior y que resultan todavía esenciales para la doctrina Coneau respecto de la Evaluación Institucional. Los mismos pueden resumirse de esta manera: la autonomía es constitutiva de la Universidad Argentina, por lo tanto no hay, al menos teóricamente, un modelo de universidad que resuma la calidad institucional y son las propias instituciones las que deben generar "su modelo institucional" y contra este debe medirse la institución real. Está claro que no hay evaluación posible sin criterios de referencia, sin estándar; evaluar es, también, comparar. Esto es así porque, como todos sabemos, el concepto de calidad es relativo, en tanto se establece en relación a un otro externo a aquello que es evaluado.

La evaluación es una operación que requiere un modelo de calidad previamente establecido; es decir, una configuración de relaciones de sentido a las que referiremos aquello que veamos en las instituciones durante el proceso evaluativo. Por lo tanto, el modelo de calidad debe construirse mediante un consenso respecto de aspectos objetivos, la metodología con que se incorpora la información, y relativos, la constitución del paradigma de calidad con el que se comparará la institución a evaluar.

Ahora, si la calidad de la institución real resulta de la comparación de su situación con el proyecto institucional que la propia universidad se ha dado, ¿qué garantía tenemos de que la institución haya elaborado un proyecto institucional de calidad, si no sabemos, no sabe tampoco la institución o, al menos no da cuenta de ello, contra qué criterios confrontar su proyecto institucional?

Cuando la Coneau, en 2006, concluye finalmente lo que fueron varios intentos de revisión de sus prácticas metodológicas respecto de las evaluaciones institucionales, y aprueba el documento “Orientaciones para el Proceso de Autoevaluación Institucional" no incluyó criterios que pudieran servir de referencia, y vuelve a dejar en claro que:

el principio rector de los Lineamientos permanece vigente, esto es, que la evaluación institucional tiene como fin primordial el mejoramiento de la calidad de las instituciones que brindan formación universitaria. Asimismo, el respeto irrestricto de la autonomía universitaria pone en valor la singularidad y la heterogeneidad de los proyectos institucionales que desarrollan las instituciones universitarias. (Coneau, 2006, p. 2)

En 2011 la Coneau dictó la Resolución № 382 aprobando un documento al que llamó: Criterios y procedimientos para la evaluación externa. Así y todo, los criterios anunciados son "un esquema analítico para uso del Comité de Pares", 
"una serie de indicaciones para el análisis" que vuelven a referenciarse en el Proyecto Institucional (p. 5). Si bien se incorporan el "contexto regional y local" y la historia de la institución como aspectos a tener en cuenta, están expresamente separados del Proyecto Institucional que sigue siendo el único referente con el que contrastar la situación de la universidad en el momento de la evaluación.

Esta insistencia se da a pesar de que todos los procesos de revisión, incluidos los anteriores que no concluyeron en algún nuevo instructivo o documento, estuvieron motivados por cuestiones verificadas en las Autoevaluaciones y en los Informes de Evaluación Externa. Las primeras no sólo presentaban modelos metodológicos diversos, a veces incongruentes, o construidos meramente por yuxtaposición de partes $\mathrm{y}$, por lo tanto, tenían estructuras diferentes, sino que en muchos casos ni siquiera se hacían cargo de la información dura referida a la institución.

Tenemos aquí una evidente consecuencia de los límites que la Ley de Educación Superior 24.521 impuso a los procesos de Evaluación Externa. Aquella norma se proponía impulsar una política de evaluación del tipo que hemos llamado de “Calificación" supone que el control de pertinencia no corresponde a la agencia de evaluación sino exclusivamente a aquel. Curiosamente, este parecer se introduce mediante el procedimiento de exaltación de la autonomía.

En este marco aparecen los problemas de la evaluación externa tal como está planteada en nuestro sistema universitario. Por un lado, el proyecto institucional aparece como una expresión de puro deseo cuya vinculación con la sociedad y con la función social que históricamente (es decir, en su contexto concreto) tal o cual universidad debe cumplir, resulta aleatoria; es una función auto-asignada lo que contradice el carácter público de la institución que la misma legislación establece. Por otra parte, los evaluadores terminarán aplicando, tanto a la situación de la universidad real, como a su proyecto institucional criterios de calidad propios, no explícitos, quizás ni siquiera coherentes entre sí, derivados de su propia "imagen modelo" de universidad o de la simple aplicación de la razonabilidad de la que sean capaces.

En este contexto la evaluación institucional se torna difusa e impredecible en sus resultados y en las posibilidades de aprovechamiento ulterior. El objetivo de mejoramiento, sin el componente de pertinencia, se diluye y las motivaciones de los actores internos lautoridades, grupos de poder, sectores internos en los claustros, etc.) cobran protagonismo sin volverse explícitas, las "imágenes de universidad" se superponen y se debaten sin haberse mostrado primero y la lectura del conjunto no orgánico, que termina siendo la autoevaluación, ofrece muy pocas posibilidades de producir en los evaluadores externos, un sentido coherente y a la vez aceptable

4. "Las políticas que se proponen utilizar la evaluación como instrumento para calificar "productos" pretenden promover la competencia entre instituciones y programas estimulando al público (clientes) mediante la exposición de una jerarquización entre las ofertas disponibles". (Pérez Rasetti, 2010) 
por la comunidad evaluada cuyos integrantes alientan, íntimamente, expectativas diferentes respecto del resultado.

La necesidad de anclar todo esto en un marco de pertinencia, es decir, un marco de referencia legitimado social y públicamente (en el sentido de "lo público"), objetivado (es decir, puesto afuera de los sujetos intervinientes), producto ly permítaseme esta audacial de una heteronomía necesaria y justificatoria de la existencia de una institución pública, debe ser resuelta a partir de la consideración elemental de que la universidad no es un ente natural, sino que, como dijo Kant (2004) ya hace muchos años, es una creación humana ("de la razón") y como, comentando aquel texto del filósofo alemán, señaló Derridá (1984), la creación de una universidad no es un hecho universitario, sino político y eso, decimos nosotros, la marca profundamente respecto de su función social.

El modelo es la imagen que de sí misma construyó la universidad en su etapa de proyecto, o la que fue buscando a través de su historia con las rectificaciones e incluso refundaciones que se hubiere dado.

La calidad sólo puede medirse desde afuera, con pretensión de objetividad (es decir, más allá de los sujetos institucionales) en términos de coherencia y para hacerlo deben conocerse los dos términos constitutivos de una relación coherente: el modelo y su realización. Lo que estamos pensando debe ajustarse a proporcionarle a cada institución instrumentos técnicos que le permitan identificar con la mayor precisión y capacidad de objetivación posible “su modelo" y el estado de las cosas, la realización de ese modelo en términos históricos.

La legitimidad de la evaluación externa necesita de una autoevaluación que proporcione los, por así llamarlos, “criterios o estándares" propios de cada institución. En nuestro contexto la evaluación "debe ser" la identificación de esos estándares implícitos en el proyecto institucional, en tanto respuesta a la función social de cada universidad concreta y el reconocimiento de la distancia que la realidad institucional representa respecto de ese modelo. "Mejorar", objetivo de la evaluación institucional, debería implicar ajustar ese modelo a la realidad histórica y contextual y recorrer un camino que achique la distancia entre el modelo revalidado y la realidad institucional. La evaluación debe hacerse en referencia a la función social de la universidad incorporada en el Proyecto Institucional en tanto este es respuesta actualizada y puesta en historia, de aquella.

\section{Referencias}

ARGENTINA. Ley de Educación Superior N²4.521. Disponible en: <http://portales. educacion.gov.ar/spu/legislaciones/ley-n_24-521/>

BROTO, Adriana. El Programa de Fortalecimiento de las Carreras de Grado. En: PUGLIESE, Juan Carlos (Editor). Políticas de Estado para la Universidad Argentina. 
Balance de una gestión en el nuevo contexto nacional e internacional. Secretaría de Políticas Universitarias. Ministerio de Educación, Ciencia y Tecnología. Buenos Aires, 2003.

CAMOU, Antonio. Los juegos de la evaluación universitaria en Argentina. Notas sobre las interacciones conflictivas entre Estado y universidad. En: CAMOU, Antonio, KROSTCH, Pedro; PRATI, Marcelo: Evaluando la evaluación. Prometeo, Buenos Aires, 2007.

CASTRO, Florencia. Críticas de las Universidades Nacionales, Secretaría de Políticas Universitarias, Mimeo. (Síntesis de las críticas que realizaron las autoridades de las universidades nacionales durante el proceso de debate de la LES, según los archivos de la Secretaría de Políticas Universitarias, ME, Argentina), 2005.

CIN (Consejo Interuniversitario Nacional). Conclusiones de las Jornadas de Reflexión sobre la Educación Superior en Argentina. Jornadas de Reflexión sobre la Educación Superior. Horco Molle, Tucumán, 2003.

Lineamientos para una nueva Ley de Educación Superior; Jornadas de Reflexión sobre la Ley universitaria, Vaquerías, 2008.

CONEAU. Avances de la Gestión desde la evaluación institucional. Buenos Aires, 2011.

Documentos relacionados con procesos de evaluación 1997/2012. Disponible en: <http://www.coneau.gov.ar> . Lineamientos para la Evaluación Institucional, Buenos Aires, 1997. Observaciones para el Procesos de Autoevaluación Institucional, Buenos Aires, 2006.

DEL BELLO, Juan Carlos; BARSKY, Osvaldo; GIMENEZ, Graciela. La universidad privada en Argentina. Libros del Zorzal, Buenos Aires, 2007.

GARCIA DE FANELLI, Ana María. Estudios de posgrado en la Argentina: una visión desde los posgrados en Ciencias Sociales. CEDES, Buenos Aires, 2000.

GUERRINI, Victoria; JEPENSSEN Cynthia; NELSON, Alejandra. Diagnóstico y perspectiva de los estudios de Posgrado en Argentina. IESALC-UNESCO/SPU, Buenos Aires, 2004.

KROTSCH, Pedro. El proceso de formación e implementación de las políticas de evaluación de la calidad en la Argentina. En: KROTSCH, Pedro: La universidad cautiva, Ediciones Al Margen, Buenos Aires, 2002.

MARQUIS, Carlos. Posgrados y Políticas Universitarias. Consideraciones sobre el caso argentino. Revista Argentina de Educación Superior. Año I, 2009.

NOSIGLIA, María Catalina; MARQUINA, Mónica. El tratamiento parlamentario de la Ley de Educación Superior. En: PAVIGLIANITI, Norma; NOSIGLIA, María Catalina y MARQUINA, Mónica. Recomposición neoconservadora. Lugar afectado: la universidad. Miño y Dávila Editores, Buenos Aires, 1996.

PAVIGLIANITI, Norma; NOSIGLIA, Catalina; MARQUINA, Mónica. Recomposición neoconservadora. Lugar afectado: la universidad. Miño y Dávila Editores, Buenos Aires, 1996. 
. La autonomía y las formas de gobierno universitario en los anteproyectos y el proyecto de ley de educación superior elaborados por el poder ejecutivo nacional. En: __ _. NOSIGLIA, María Catalina y MARQUINA, Mónica: Recomposición neoconservadora. Lugar afectado: la universidad. Miño y Dávila Editores, Buenos Aires, 1996.

PUGLIESE, Juan Carlos. Universidades y Estado: una asociación para responder a los desafíos del cambio. Políticas de Estado para las Universidades. Ministerio de Educación, Ciencia y Tecnología. Buenos Aires, 2004. En . Políticas de Estado para la Universidad Argentina. Balance de una gestión en el nuevo contexto nacional e internacional. Secretaría de Políticas Universitarias. Ministerio de Educación, Ciencia y Tecnología. Buenos Aires, 2003. STUBRIN, Adolfo. Calidad universitaria. Evaluación y acreditación en la educación superior latinoamericana. Eudeba/EdicionesUNL, Santa Fe/Buenos Aires, 2010. PEREZ RASETTI, Carlos. Evaluación y Políticas Públicas: los sentidos de la evaluación. En: Revista Gestión Universitaria. V.3. n.1. Nov. 2010. Disponible en: $<$ http://www.gestuniv.com.ar/gu_07/v3n1a3.htm>

. Compromiso del Estado con las Universidades Nacionales. Políticas de Mejoramiento de las Capacidades Institucionales. Documento de la Secretaría de Políticas Universitarias, Jornadas de Reflexión CIN, Mar del Plata, 2005.

Recebido em outubro de 2012

Aprovado em janeiro de 2013

Carlos Pérez Rasetti é profesor en Humanidades, especialidad Letras, IUSCUniversidad Nacional del Sur, Argentina. Profesor Investigador de la Universidad Nacional de la Patagonia Austral (Arg.). Director de Maestría en Educación Superior en la Universidad Nacional de la Matanza (Arg.). Email: prasettiđciudad.com.ar 